\title{
Contracting-on-Average Baker Maps
}

\author{
by \\ Michał RAMS \\ Presented by Andrzej LASOTA
}

Summary. We estimate from above and below the Hausdorff dimension of SRB measure for contracting-on-average baker maps.

1. Introduction. One of the main open problems in the dimension theory of dynamical systems is how to work with non-conformal systems, i.e. systems with two (or more) different negative Lyapunov exponents or with two (or more) different positive Lyapunov exponents. The simplest such systems are solenoids, and it is for them that some results are known (cf. [B], [S2], [SS], [RS]).

These results were achieved by considering a projection of the system in the direction of maximal contraction. The resulting (non-invertible) hyperbolic systems are called baker maps. They are of independent interest and were studied as well: see for example [S1], [T], [R1].

In all these papers a transversality condition was assumed, first introduced by Pollicott and Simon [PS] in the context of one-parameter families of iterated function systems. This condition is open and it is easy to show examples of systems satisfying it. For some regions in parameter space, the transversality condition is satisfied on a dense subset, as shown by Bothe [B].

In this paper we consider a generalisation of baker maps: contracting-onaverage baker maps. Those are non-uniformly hyperbolic systems. However, we show the same dimension estimates for the SRB measures of COA baker maps as are known for standard baker maps. The upper bound does not

2000 Mathematics Subject Classification: Primary 28A78, 37D25.

Key words and phrases: baker map, Hausdorff dimension, SRB measure, transversality condition.

Supported by Polish KBN Grant No 2P0 3A 03425. 
require any additional assumptions. To obtain the lower bound we need the transversality condition to be satisfied.

\section{Geometry of contracting-on-average baker maps}

2.1. Definition of contracting-on-average baker maps. Let us start from some notations. We are going to use three symbolic spaces:

$$
\begin{aligned}
\Sigma_{-} & =\{0, \ldots, k-1\}^{-\mathbb{N} \cup\{0\}}, \\
\Sigma_{+} & =\{0, \ldots, k-1\}^{\mathbb{N}}, \\
\Sigma & =\Sigma_{-} \times \Sigma_{+}=\{0, \ldots, k-1\}^{\mathbb{Z}} .
\end{aligned}
$$

We define a map $\sigma$ acting on $\Sigma_{-}$and on $\Sigma$ by

$$
(\sigma \omega)_{i}=\omega_{i-1} .
$$

On $\Sigma_{+}$we define $k$ maps $\sigma_{i}$ as follows:

$$
\sigma_{i}\left(\omega_{1} \omega_{2} \ldots\right)=i \omega_{1} \omega_{2} \ldots, \quad i=0, \ldots, k-1 .
$$

Finite sequences of symbols from $\{0, \ldots, k-1\}$ will be denoted by $\omega^{n}=$ $\omega_{1} \ldots \omega_{n}$. By $\omega^{-n}$ we will denote $\omega^{n}$ written backwards, i.e. $\omega^{-n}=\omega_{n} \ldots \omega_{1}$.

We will consider maps of the form

$$
f(x, y)=(g(x), h(x, y))
$$

acting on $S^{1} \times \mathbb{R}$.

We demand that $g$ is a $k-1 C^{2}$ orientation preserving expanding map of the circle, i.e. $g$ is $C^{2}, g^{\prime}>1$ everywhere and every point has precisely $k$ preimages under $g$. We parametrise the circle as the interval $(0,1]$, where 1 is one of the fixed points of $g$. We assume $g$ to be $C^{2}$ except possibly at points $x \in g^{-1}(1)$ and to be continuous and have left and right derivative everywhere. Similarly, we assume $h$ to be $C^{2}$ except on the lines $\left\{x \in g^{-1}(1)\right\}$.

We will also assume that the map $f$ has uniformly bounded first and second order derivatives in its whole domain (again except possibly on the lines $\left.\left\{x \in g^{-1}(1)\right\}\right)$ and that $\partial_{2} h$ is bounded away from zero. We denote the iterations of $f$ by $f^{n}(x, y)=\left(g^{n}(x), h_{n}(x, y)\right)$.

Given $x$, the supremum of $\left|\partial_{2} h(x, \cdot)\right|$ will be denoted by $\Phi(x)$; we assume that

$$
|\Phi(x)|<\left|g^{\prime}(x)\right|
$$

Let

$$
L=\exp \left(\int_{S^{1}} \log \Phi(x) d \nu_{-}(x)\right) .
$$

DeFinition 2.1. The map (2.1) satisfying the smoothness assumptions above will be called a contracting-on-average (COA) baker map if $L<1$. 
2.2. One-dimensional dynamics of $g$. The results of this subsection are a standard application of the thermodynamical formalism to expanding maps. An introduction to the thermodynamical formalism can be found in Bowen's book [Bo]. The way of applying it to expanding maps is explained (in a slightly different situation) in the fourth chapter of Falconer's book [F], so we will omit the detailed proofs.

There are $k$ intervals of the form $(a, b]$ that are mapped by $g$ onto $(0,1]$ in a bijective way. These intervals are pairwise disjoint and form a covering of $(0,1]$. We will denote them by $G_{0}, \ldots, G_{k-1}$. The branch of $g^{-1}$ moving $(0,1]$ onto $G_{i}$ will be denoted by $g_{i}$.

Similarly, we have $k^{n}$ intervals of the form $(a, b]$ that are mapped by $g^{n}$ onto $(0,1]$ in a bijective way. We will write $G_{\omega^{n}}=g_{\omega_{1}}\left(G_{\omega_{2} \ldots \omega_{n}}\right)$. The corresponding branch of $g^{-n}$ will be denoted by $g_{\omega^{-n}}=g_{\omega_{n}} \circ \cdots \circ g_{\omega_{1}}$.

We introduce a symbolic expansion on $(0,1]$ in the following way:

$$
\pi_{i}(x)=\left\{j ; g^{i}(x) \in G_{j}\right\}, \quad i \geq 0 .
$$

In other words,

$$
x=\bigcap_{n=0}^{\infty} G_{\pi_{0}(x) \ldots \pi_{n}(x)} .
$$

Let $\tau(x)=\ldots \pi_{n}(x) \ldots \pi_{1}(x) \pi_{0}(x)$. The map $\tau:(0,1] \rightarrow \Sigma_{-}$is bijective and almost onto (the set $\Sigma_{-} \backslash \tau((0,1])$ is countable). Moreover, it is a conjugation between $g$ acting on $(0,1]$ and $\sigma$ acting on $\Sigma_{-}$.

For any function $\psi:(0,1] \rightarrow \mathbb{R}$ define $S^{n} \psi(x)=\sum_{i=0}^{n-1} \psi\left(g^{i}(x)\right), \quad S_{-}^{n} \psi\left(\omega^{n}\right)=\inf _{G_{\omega^{n}}} S^{n} \psi(x), \quad S_{+}^{n} \psi\left(\omega^{n}\right)=\sup _{G_{\omega^{n}}} S^{n} \psi(x)$.

The following lemma is equivalent to Proposition 4.1 in $[\mathrm{F}]$.

Lemma 2.2. Let $\psi:(0,1] \rightarrow \mathbb{R}$ be Lipschitz on every $G_{i}$. Then $\max S^{n} \psi(\cdot)-\min S^{n} \psi(\cdot)$ is bounded inside any $G_{\omega^{n}}$; the bound is uniform with respect to both $n$ and $\omega^{n}$.

Hence, $S_{+}^{n} \psi-S_{-}^{n} \psi<K$ with $K$ depending only on $\psi$.

The Lasota-Yorke theorem states that there exists a unique absolutely continuous $g$-invariant probability measure $\nu_{-}$on $(0,1]$ and its density is bounded away from zero and infinity. As every $g_{i}$ is $C^{2}$, if we take $\left.\nu_{-}\right|_{G_{\omega}}$ for any $\omega^{n}$, iterate it $n$ times under $g$ and normalise, the resulting measure will still have its density uniformly bounded away from zero and infinity. Hence,

$$
K^{-1} \leq\left.\frac{1}{\nu_{-}\left(G_{\omega^{n}}\right)} d \nu_{-}\right|_{G_{\omega^{n}}} d\left(\nu_{-} \circ g_{\omega^{-n}}\right) \leq K
$$

for some $K$ independent of $\omega$ and $n$. 
The image of $\nu_{-}$under $\tau$ is a shift-invariant probability measure on $\Sigma_{-}$ that will be denoted by $\mu_{-}$. It is the equilibrium measure for the potential $\phi(\omega)=-\log g^{\prime}\left(\tau^{-1}(\omega)\right)$, hence it is ergodic.

For $\psi$ as in Lemma 2.2 set

$D(\psi, K, \varepsilon, n)=\left\{x ; n(1-\varepsilon) \int \psi d \nu_{-}-K \leq S^{n} \psi(x) \leq n(1+\varepsilon) \int \psi d \nu_{-}+K\right\}$.

The central limit theorem for equilibrium measures (Theorem 1.27 in [Bo]) implies the following lemma.

Lemma 2.3. Let $\psi$ be as in Lemma 2.2. Fix $\varepsilon>0$. For any $\delta>0$ there exists $K>0$ such that

$$
\sum_{n=1}^{\infty}\left(1-\nu_{-}(D(\psi, K, \varepsilon, n))\right)<\delta .
$$

In particular, for any positive $\varepsilon$ and for $\nu_{-}$-almost every $x \in(0,1]$ there exists $K_{1}$ such that

$$
K_{1}^{-1} \lambda_{-}^{n(1+\varepsilon)}<\left|G_{\tau^{n}(x)}\right|<K_{1} \lambda_{-}^{n(1-\varepsilon)},
$$

where

$$
\lambda_{-}=\exp \left(-\int_{S^{1}} \log g^{\prime}(x) d \nu_{-}(x)\right) .
$$

2.3. Geometry and dynamics of $f, S R B$ measure. The measure $\mu_{-}$is $\sigma$-invariant. Hence there exists a unique $\sigma$-invariant measure on $\Sigma$ for which $\mu_{-}$is the marginal distribution on $\Sigma_{-}$. We will denote this measure by $\mu$ and let $\mu_{+}$be the marginal distribution of $\mu$ on $\Sigma_{+}$. As $\mu_{-}$is ergodic, so is $\mu$.

Fix a small positive $\varepsilon$ and let $\omega$ be a sequence from $\Sigma_{+}$such that for some $K$ and for all $n$,

$$
S^{n} \log \Phi\left(\omega^{-n}\right)<n(1-\varepsilon) \log L+K .
$$

Lemma 2.3 guarantees us plenty of such $\omega$. Consider the sequence of curves

$$
f^{n}\left(G_{\omega^{-n}} \times\{0\}\right) .
$$

They are graphs of $C^{2}$ functions $y=\Lambda_{n, \omega}(x)$.

Proposition 2.4. Assume that (2.5) is satisfied for some $\omega \in \Sigma_{+}$. Then the sequence $\Lambda_{n, \omega}$ converges uniformly in $C^{2}$ to some $\Lambda_{\omega}$. Moreover, the first two derivatives of $\Lambda_{\omega}$ are uniformly bounded independently of $\omega$. The limit does not change if in (2.6) we replace $G_{\omega^{-n}} \times\{0\}$ by $G_{\omega^{-n}} \times\{t\}$ for any $t$.

Proof. We have

$$
\left.\Lambda_{n+1, \omega}(x)-\Lambda_{n, \omega}(x)=h_{n}\left(g_{\omega^{n}}(x), h\left(g_{\omega_{n+1}}, 0\right)\right)\right)-h_{n}\left(g_{\omega^{n}}(x), 0\right) .
$$


By the choice of $\omega,\left|\partial_{2} h_{n}\right|$ is not greater than $e^{K} L^{n(1-\varepsilon)}$. As this is a summable series and $|h(\cdot, 0)|$ is uniformly bounded, $\Lambda_{n, \omega}(x)$ is a Cauchy sequence in sup norm.

Of course, if we used $f^{n}\left(G_{\omega^{-n}} \times\{t\}\right)$ (the graphs of functions that we will denote by $\left.\Lambda_{n, \omega}^{t}\right)$, the limit would be the same, as

$$
\left\|\Lambda_{n, \omega}^{t}-\Lambda_{n, \omega}\right\|=\left\|h_{n}(\cdot, t)-h_{n}(\cdot, 0)\right\| \leq t \cdot e^{K} L^{n(1-\varepsilon)} \rightarrow 0 .
$$

To estimate the derivatives of $\Lambda_{\omega}$ note that $f$ may be locally expanding in the vertical direction, but the expansion in the horizontal direction is stronger (by a uniform constant). We can thus write $f$ locally as the sum of a linear map $f_{0}(x, y)=\left(x_{0}+M\left(x-x_{0}\right), y_{0}+m\left(y-y_{0}\right)\right.$ ) (with $M / m$ uniformly greater than 1) and a nonlinear distortion term $f_{1}$ (with first and second order derivatives uniformly bounded). Hence,

$$
\left|\Lambda_{n+1, \omega}^{\prime}\right| \leq \frac{m}{M} \max \left|\Lambda_{n, \omega}^{\prime}\right|+C
$$

and

$$
\left|\Lambda_{n+1, \omega}^{\prime \prime}\right| \leq \frac{m}{M^{2}} \max \left|\Lambda_{n, \omega}^{\prime \prime}\right|+C,
$$

thus the first and second derivatives of $\Lambda_{\omega}$ exist and are uniformly bounded (note that the bounds do not depend on $K$ or $\varepsilon$; we only need to know that $\Lambda_{n, \omega}$ converges).

The curves $\Lambda_{\omega}$ will be called the leaves of the map $f$. We proved the existence of the leaves for some $\omega$ given by (2.5). Denote by $\widetilde{\Sigma}_{+}$the set of $\omega$ for which leaves exist. As the image of a leaf under $f$ is a union of $k$ leaves,

$$
\widetilde{\Sigma}_{+} \subset \bigcup_{i=0}^{k-1} \sigma_{i}\left(\widetilde{\Sigma}_{+}\right) .
$$

Henceforth we will concentrate on the points of the form $\left(x, \Lambda_{\omega}(x)\right)$ for $\omega \in \widetilde{\Sigma}_{+}$. The natural symbolic space for such points is $\widetilde{\Sigma}=\Sigma_{-} \times \widetilde{\Sigma}_{+} \subset \Sigma$. Let us denote this expansion by $\widetilde{\tau}(x, y)$, i.e. $\widetilde{\tau}:(0,1] \times \mathbb{R} \rightarrow \widetilde{\Sigma}$. This map is not uniquely defined - intersection points of leaves will have more than one symbolic expansion (corresponding to their history under iteration of $f$ ). The map $\widetilde{\tau}$ conjugates $f$ with $\sigma$ acting on $\widetilde{\Sigma}$ as a subset of $\Sigma$. By (2.7), $\widetilde{\Sigma}$ is $\sigma$-invariant.

Note here a relation between the measures $\mu_{-}$and $\mu_{+}$. Denote by $C_{-}\left(\omega^{n}\right)$ the set of all sequences from $\Sigma_{-}$ending with $\omega^{n}$ and by $C_{+}\left(\omega^{n}\right)$ set of all sequences from $\Sigma_{+}$beginning with $\omega^{n}$. Then by invariance of $\mu$ under $\sigma$,

$$
\mu_{-}\left(C_{-}\left(\omega^{n}\right)\right)=\mu_{+}\left(C_{+}\left(\omega^{n}\right)\right) .
$$

Lemma 2.3 implies that $\widetilde{\Sigma}$ has positive $\mu$-measure. By ergodicity of $\mu$, we have $\mu(\widetilde{\Sigma})=1$. 
We can project $\mu$ back from the symbolic space by $\pi=\widetilde{\tau}^{-1}$; the resulting measure will be denoted by $\nu$. It is the SRB measure for our system and we will work with it in the rest of the paper. For $\nu$ we compute the Lyapunov exponent of the map $f$ :

$$
\log \lambda=\int \log \left|\partial_{2} h(x, y)\right| d \nu(x, y)
$$

(it is the Lyapunov exponent in the vertical direction; the one in the horizontal direction equals $-\log \lambda_{-}$and we need only $g$ and $\nu_{-}$to compute it).

We need to introduce our last geometric assumption: transversality.

Definition 2.5. If there exists $b>0$ such that for any two leaves $\Lambda_{\omega}$ and $\Lambda_{\widetilde{\omega}}$ with $\omega_{1} \neq \widetilde{\omega}_{1}$ we have

$$
\left|\Lambda_{\omega}(x)-\Lambda_{\widetilde{\omega}}(x)\right|+\left|\Lambda_{\omega}^{\prime}(x)-\Lambda_{\widetilde{\omega}}^{\prime}(x)\right|>b
$$

then we say that the baker map satisfies the transversality condition.

The name comes from the fact that the condition implies that two such leaves have only a uniformly bounded number of intersections and those intersections are transversal. As the transversality of intersections between leaves is preserved under iteration of $f$, the transversality condition implies that all the leaf intersections are transversal (but not conversely).

The transversality condition is open and we can give a family of examples of baker maps satisfying it.

EXAmple 2.6. Consider a linear baker map of the form $g(x)=k x$ $(\bmod 1)$ and $h(x, y)=h_{1}^{j} x+h_{2}^{j} y$, with $h_{1}^{j}$ and $h_{2}^{j}$ depending only on $j=\lfloor k x\rfloor$. For such a map, $\Lambda_{\omega}(x)=b(\omega)+a(\omega) x$, where

$$
a(\omega)=\sum_{i} k^{-i} S^{i} h_{2}\left(\omega^{-i+1}\right) h_{1}\left(\omega^{-i}\right) .
$$

In other words, $a(\omega)$ is the limit point of the iterated function system of the maps

$$
f_{j}: x \mapsto h_{2}^{j} k^{-1} x+h_{1}^{j}
$$

and such a system will satisfy the strong separation condition if $\sum h_{2}^{j}<k$, for properly chosen $h_{1}^{j}$. The strong separation condition for this system implies that $a(\omega)$ and $a(\widetilde{\omega})$ will differ at least by a constant if $\omega_{1} \neq \widetilde{\omega}_{1}$, hence the transversality follows.

We can now formulate our main theorem. We define

$$
s=\frac{\log \lambda_{-}}{\log \lambda} .
$$

THEOREM 2.7. For any contracting-on-average baker map the upper Hausdorff dimension of its SRB measure is not greater than $\min (2,1+s)$. 
If the map satisfies the transversality condition, then the upper and lower Hausdorff dimensions of the SRB measure are equal to $\min (2,1+s)$.

\section{Proof of Theorem 2.7}

3.1. Upper bound for Hausdorff dimension. We may freely assume $s<1$, otherwise the assertion is empty. We fix some small positive $\varepsilon$. We denote by $W_{K}$ the set of points $(x, y) \in \pi(\widetilde{\Sigma}) \cap\{y \in[-K, K]\}$ for which for all $n$,

$$
n(1+\varepsilon) \log \lambda-\log K<\sum_{i=0}^{n-1} \log \left|\partial_{2} h_{i}(x, y)\right|<n(1-\varepsilon) \log \lambda+\log K
$$

and (2.4) holds.

For increasing $K, \nu\left(W_{K}\right)$ will increase to 1 by Lemma 2.3. We choose some large $K$.

We have to check how some iteration $f^{n}$ ( $n$ large) behaves in the neighbourhood of a point $(x, y) \in W_{K}$. We know that (3.1) holds at $(x, y)$ and would like to find some neighbourhood of this point where it holds as well (possibly with worse $K$, but at most worse by a multiplicative constant).

We will take this neighbourhood $U$ in the form of a rectangle $U=I_{1} \times I_{2}$, $x \in I_{1}, y \in I_{2}$.

LEMma 3.1. There exists $d_{K}$ such that for all $n$, if the rectangle $U=$ $I_{1} \times I_{2}$ has nonempty intersection with $W_{K}$ where $I_{1}=G_{\omega^{n}}$ and $\left|I_{2}\right|<d_{K}$ then

$$
n(1+\varepsilon) \log \lambda-\log K_{0}<\sum_{i=0}^{n-1} \log \left|\partial_{2} h\left(f^{i}(\cdot)\right)\right|<n(1-\varepsilon) \log \lambda+\log K_{0}
$$

for all points in $U$, with $K_{0}$ depending on $K$ and $\varepsilon$ but not on $n$.

Proof. The map $f$ is $C^{2}$ and its derivative $\left|\partial_{2} h\right|$ is bounded away from zero. Hence, its logarithm is $C^{1}$ with bounded derivative and the condition we need is that the sum of the diameters of the first $n$ images of $U$ under $f$ is uniformly bounded:

$$
\sum_{j=0}^{n-1}\left|f^{j}(U)\right| \leq c(K)
$$

Any image of $U$ is an approximate rectangle: the images of the vertical intervals are vertical intervals while the horizontal intervals are mapped onto approximately horizontal lines (graphs of functions with uniformly bounded derivative; check the proof of Proposition 2.4). Hence

$$
\left|f^{j}(U)\right| \leq c\left|g^{j}\left(I_{1}\right)\right|+\inf _{x \in I_{1}}\left|h_{j}\left(x, I_{2}\right)\right| .
$$


The first part of the sum (3.2) is easy to estimate: $\sum_{j=0}^{n-1}\left|g^{j}\left(G_{\omega^{n}}\right)\right|$ is uniformly bounded and the bound depends neither on $n$ nor on $\omega^{n}$. To estimate the second part, let $(x, t) \in U \cap W_{K}$ and consider $z_{j}=\left|h_{j}\left(\{x\} \times I_{2}\right)\right|$. We have

$$
z_{j} \leq d_{K} \max _{y \in I_{2}}\left|\partial_{2} h_{j}(x, y)\right| .
$$

We can use the Leibniz formula for the derivative to get

$$
\log \left|\frac{\partial_{2} h_{j}(x, y)}{\partial_{2} h_{j}(x, t)}\right| \leq c \sum_{i=0}^{j-1}\left|h_{i}(x, y)-h_{i}(x, t)\right| \leq c \sum_{i=0}^{j-1} z_{i}
$$

and using (3.1) one gets bounds like those in Lemma 4.3 from [R2].

Hence, $\sum_{i=0}^{n-1} z_{i}$ is bounded and the bound does not depend on $n$.

We cover $W_{K}$ by rectangles of the form $G_{\omega^{n}} \times\left[j d_{K},(j+1) d_{K}\right]$ and take the image of this family under $f^{n}$. We get a family of approximately horizontal strips with the following properties. First, as stated above, their width is not greater than $K_{0} \lambda^{n(1-\varepsilon)}$. Second, there are at most $\left(2 K / d_{K}\right) \cdot K \lambda_{-}^{-n(1+\varepsilon)}$ strips. Third, their union contains $f^{n}\left(W_{K}\right)$, hence its measure $\nu$ is at least $\nu\left(W_{K}\right)$.

Cutting those strips into approximate squares $\left\{E_{i}\right\}$ we get a cover of a set of measure at least $\nu\left(W_{K}\right)$ with $2 K^{2} K_{0}^{-1} \lambda_{-}^{-n(1+\varepsilon)} \lambda^{-n(1-\varepsilon)}$ squares of size $K_{0} \lambda^{n(1-\varepsilon)}$. If $n$ was chosen sufficiently large,

$$
\sum\left(\operatorname{diam} E_{i}\right)^{1+s(1+3 \varepsilon)}<1 .
$$

Repeating all this procedure for greater and greater $K$ and $n$ we get a family of sets of increasing measures and their finer and finer covers, satisfying (3.3). The upper limit of those sets has measure 1. We can cover this set with the union of our covers and this proves that the resulting set has zero Hausdorff measure in any dimension greater than $1+s(1+3 \varepsilon)$.

As $\varepsilon$ may be chosen arbitrarily small, we are done.

3.2. Lower bound for Hausdorff dimension. The measure $\nu$ is absolutely continuous on leaves by (2.3). Even more, by Lemma 2.2 its density varies at most by a constant along any leaf. Hence, we can write

$$
d \nu(x, y) \approx d x \cdot \sum \pi^{*} \mu_{+}(d \omega),
$$

where the sum is taken over all leaves $\Lambda_{\omega}$ passing through $(x, y)$.

Fix a small $\varepsilon$. We denote by $V_{K}$ the set of leaves $\Lambda_{\omega}$ for which for all $n$, for at least one point $x$,

$$
\begin{aligned}
n(1+\varepsilon) \log \lambda-\log K & <\sum_{i=0}^{n-1} \log \left|\partial_{2} h\left(f^{i}\left(x, \Lambda_{\omega}(x)\right)\right)\right| \\
& <n(1-\varepsilon) \log \lambda+\log K
\end{aligned}
$$


and

(3.6) $n(1+\varepsilon) \log \lambda_{-}-\log K<\mu_{+}\left(C_{+}\left(\omega^{n}\right)\right)<n(1-\varepsilon) \log \lambda_{-}+\log K$.

The formula (3.6) may be seen as dual to (2.4); it is satisfied for an arbitrarily large set of $\omega$ by Lemma 2.3. We denote by $\nu_{K}$ the restriction of $\nu$ to $V_{K}$.

Note that if $\left(x, \Lambda_{\omega}(x)\right)$ satisfies (3.5) then for all $x^{\prime},\left(x^{\prime}, \Lambda_{\omega}\left(x^{\prime}\right)\right)$ will satisfy (3.5) for slightly (by a multiplicative constant) greater $K$. Hence, we still have

$$
d \nu_{K}(x, y) \approx d x \cdot \sum \pi^{*} \mu_{+K}(d \omega)
$$

for some measure $\mu_{+K} \leq \mu_{+}$of norm close to 1 .

We are going to estimate from above the integral

$$
Z(K, \delta)=\int \nu_{K}\left(B_{\delta}(x, y)\right) d \nu_{K}(x, y)
$$

(for small $\delta$ ), where $B_{\delta}(x, y)$ is the square of side-length $\delta$ centred at $(x, y)$. By (3.7) we can write

$$
Z(K, \delta) \leq c \iiint \operatorname{Leb}\left(B_{\delta}\left(x, \Lambda_{\omega}(x)\right) \cap \Lambda_{\omega^{\prime}}\right) d x d \mu_{+K}(\omega) d \mu_{+K}\left(\omega^{\prime}\right)
$$

Set

$$
I_{\delta}\left(\omega, \omega^{\prime}\right)=\int \operatorname{Leb}\left(B_{\delta}\left(x, \Lambda_{\omega}(x)\right) \cap \Lambda_{\omega^{\prime}}\right) d x .
$$

If $\omega_{1} \neq \omega_{1}^{\prime}$, the above is easy to estimate from transversality: whenever $\Lambda_{\omega}$ and $\Lambda_{\omega^{\prime}}$ are at a distance smaller than $b / 2$, their derivatives must differ by at least $b / 2$. Hence for such pairs

$$
I_{\delta}\left(\omega, \omega^{\prime}\right) \leq \min \left(\frac{4}{b} \delta^{2}, 1\right)
$$

We will write

$$
Z(K, \delta) \leq \sum_{n} Z_{n}(K, \delta),
$$

where $Z_{n}(K, \delta)$ is the part of the integral (3.8) over pairs $\omega, \omega^{\prime}$ with first $n$ symbols identical.

Let $\omega=\eta^{n} \alpha$ and $\omega^{\prime}=\eta^{n} \beta, \alpha_{1} \neq \beta_{1}$. We will estimate not $I_{\delta}\left(\omega, \omega^{\prime}\right)$ but

$$
J_{n, \delta}(\alpha, \beta)=\sum_{\eta^{n}} I_{\delta}\left(\eta^{n} \alpha, \eta^{n} \beta\right),
$$

where the sum is taken over all $n$-digit sequences $\eta^{n}$ such that both $\Lambda_{\eta^{n} \alpha}$ and $\Lambda_{\eta^{n} \beta}$ belong to $V_{K}$. Note that

$$
d \mu_{+}\left(\tau^{n} \alpha\right) \approx \mu_{+}\left(C_{+}\left(\tau^{n}\right)\right) d \mu_{+}(\alpha)
$$

(this follows from a similar estimate for $\nu_{-}$given by Lemma 2.2). For $\mu_{+K}$ the same estimate holds, but only for $\tau^{n}$ and $\alpha$ such that $\alpha \in V_{K}$ and $\tau^{n}$ satisfies (3.6). 
We thus have

$$
Z_{n}(K, \delta) \leq \sup _{\eta^{n}} \mu_{+K}\left(C_{+}\left(\eta^{n}\right)\right) \iint J_{n, \delta}(\alpha, \beta) d \mu_{+}(\alpha) d \mu_{+}(\beta) .
$$

The union of the pairs $\left(\Lambda_{\eta^{n} \alpha}, \Lambda_{\eta^{n} \beta}\right)$ is the image of $\left(\Lambda_{\alpha}, \Lambda_{\beta}\right)$ under $f^{n}$. By assumption we keep only those branches $\eta^{n}$ of the map $f^{n}$ for which it is locally a contraction in the vertical direction (with contraction ratio not greater than $D_{1}^{+}=c K \lambda^{n(1-\varepsilon)}$ and not smaller than $\left.D_{1}^{-}=(c K)^{-1} \lambda^{n(1+\varepsilon)}\right)$ and a dilatation in the horizontal direction (with dilatation ratio between $D_{2}^{+}=K \lambda_{-}^{-n(1+\varepsilon)}$ and $D_{2}^{-}=K^{-1} \lambda_{-}^{-n(1-\varepsilon)}$ ). The square $B_{\delta}$ is the image of an approximate rectangle with horizontal side-length between $\delta / D_{2}^{+}$and $\delta / D_{2}^{-}$ and vertical side-length $\delta / D_{1}, D_{1} \in\left[D_{1}^{-}, D_{1}^{+}\right]$. Hence,

$$
J_{n, \delta}(\alpha, \beta) \leq I_{\delta / D_{1}} \cdot \frac{D_{1}}{D_{2}^{-}} \cdot D_{2}^{+} \leq D_{1}^{+} K^{2} \lambda_{-}^{-2 n \varepsilon} \min \left(\frac{4 \delta^{2}}{b\left(D_{1}^{-}\right)^{2}}, 1\right) .
$$

Substituting this into (3.10) and estimating $\sup _{\eta^{n}} \mu_{+K}\left(C_{\eta^{n}}\right)$ by $K \lambda_{-}^{n(1-\varepsilon)}$ from (3.6), we get

$$
Z_{n}(K, \delta) \leq \min \left[c(K)\left(\frac{\lambda_{-}}{\lambda}\right)^{n(1-3 \varepsilon)} \delta^{2}, c(K) \lambda_{-}^{n(1-3 \varepsilon)} \lambda^{n(1-\varepsilon)}\right] .
$$

Now, there are two cases: $s<1$ or $s \geq 1$. Assume first that $s<1$. The sequence $Z_{n}$ is first increasing (exponentially fast) and then decreasing (also exponentially fast). The sum of the series in (3.9) is thus approximately equal to the greatest element, i.e.

$$
Z(K, \delta) \leq c(K) \lambda_{-}^{n_{0}(1-3 \varepsilon)} \lambda^{n_{0}(1-\varepsilon)}
$$

for

Hence

$$
n_{0}=\frac{\log \delta-c(K)}{(1+\varepsilon) \log \lambda}
$$

$$
Z(K, \delta) \leq c(K) \delta^{1-3 \varepsilon+(1-5 \varepsilon) s}
$$

for $\delta$ small enough. By Tsujii's lemma from [T], for all $K$ the Hausdorff dimension of $\nu_{K}$ (hence, of $\nu$ as well) is not smaller than $1-3 \varepsilon+(1-5 \varepsilon) s$. As $\varepsilon$ may be chosen arbitrarily small, the assertion follows.

Let now $s \geq 1$. The sequence $Z_{n}$ is then nonincreasing (even decreasing when $s>1$ ) for $n<n_{0}$, so we can estimate the sum in (3.9) by $n_{0} Z_{0}$ :

$$
Z(K, \delta) \leq c(K) \delta^{2}|\log \delta|
$$

Now Tsujii's lemma implies that the Hausdorff dimension of $\nu_{K}$ is equal to 2.

\section{References}

[B] H. G. Bothe, The Hausdorff dimension of certain solenoids, Ergodic Theory Dynam. Systems 15 (1995), 449-474. 
[Bo] R. Bowen, Equilibrium States and the Ergodic Theory of Anosov Diffeomorphisms, Springer, Heidelberg, 1975.

[F] K. Falconer, Techniques in Fractal Geometry, Wiley, Chichester, 1997.

[PS] M. Pollicott and K. Simon, The Hausdorff dimension of $\lambda$-expansions with deleted digits, Trans. Amer. Math. Soc. 347 (1995), 967-983.

[R1] M. Rams, Absolute continuity of the SBR measure for non-linear fat baker maps, Nonlinearity 16 (2003), 1649-1655.

[R2] -, Dimension estimates for invariant measures of contracting-on-average iterated function systems, preprint.

[RS] M. Rams and K. Simon, Hausdorff and packing measure for solenoids, Ergdic Theory Dynam. Systems 23 (2003), 273-292.

[S1] K. Simon, Hausdorff dimension for non-invertible maps, ibid. 13 (1993), 199-212.

[S2] - The Hausdorff dimension of the general Smale-Williams solenoid, Proc. Amer. Math. Soc. 125 (1997), 1221-1228.

[SS] K. Simon and B. Solomyak, Hausdorff dimension for horseshoes in $\mathbb{R}^{3}$, Ergodic Theory Dynam. Systems 19 (1999), 1343-1363.

[T] M. Tsujii, Fat solenoidal attractors, Nonlinearity 14 (2001), 1011-1027.

Michał Rams

Institute of Mathematics

Polish Academy of Sciences

Śniadeckich 8

00-950 Warszawa, Poland

E-mail: rams@impan.gov.pl

Received June 1, 2006;

received in final form November 7, 2006 